Journal of Applied Pharmaceutical Science Vol. 4 (10), pp. 007-011, October, 2014

Available online at http://www.japsonline.com

DOI: $10.7324 / J A P S .2014 .401002$

ISSN 2231-3354 (cc) BY-NC-SA

\title{
Triterpenes and Sterol from Artocarpus ovatus
}

\author{
Consolacion Y. Ragasa ${ }^{1.2}$, Jocelyn L. Caro ${ }^{2}$, Chien-Chang Shen ${ }^{3}$ \\ ${ }^{1}$ Chemistry Department, De La Salle University Science \& Technology Complex Leandro V. Locsin Campus, Biñan City, Laguna, Philippines. \\ ${ }^{2}$ Chemistry Department De La Salle University, 2401 Taft Avenue, Manila, 1004, Philippines. \\ ${ }^{3}$ National Research Institute of Chinese Medicine, 155-1, Li-Nong St., Sec. 2, Taipei 112, Taiwan.
}

\begin{tabular}{l} 
ARTICLE INFO \\
\hline Article history: \\
Received on: 12/08/2014 \\
Revised on: 26/08/2014 \\
Accepted on: $18 / 09 / 2014$ \\
Available online: $30 / 10 / 2014$ \\
\hline
\end{tabular}

Key words:

Artocarpus ovatus, Moraceae,

triterpenes, sterol

\section{INTRODUCTION}

Artocarpus ovatus Blanco, an endemic Philippine tree, locally known as anubing is an evergreen tree growing up to 25 meters tall. A latex obtained from the tree has potential for use as a chewing gum base (Fern, 2012). The roasted seeds are eaten, while the timber which is strong and durable is used for construction (De la Cruz, 1991).

There is no reported study on the chemical constituents of A. ovatus. However, congeners of the tree have been studied for their chemical constituents and biological activities. There are about 50 species of the genus Artocarpus (Moraceae) which are sources of edible fruit and timber and are used in folk medicines. A review of the chemical constituents, biological and pharmacological activities of Artocarpus has been provided (Jagtap and Bapat, 2010). Artocarpus species are used for treatment against inflammation, malarial fever, diarrhoea, diabetes and tapeworm infection. They are rich in phenolic compounds such as flavonoids, stilbenoids, arylbenzofurons \& jacalin (Jagtap and Bapat, 2010). Compounds from Artocarpus species exhibit diverse biological activities including antibacterial, antitubercular,

\footnotetext{
* Corresponding Author

Consolacion Y. Ragasa, Chemistry Department, De La Salle University, 2401 Taft Avenue, Manila 1004 Philippines.

Email: consolacion.ragasa@dlsu.edu.ph
}

antiviral, antifungal, antiplatelet, antiarthritic, tyrosinase inhibitory and cytotoxicity (Jagtap and Bapat, 2010). This study is part of our research on the chemical constituents of the genus Artocarpus found in the Philippines.

We earlier reported the isolation of friedelinol, squalene, $\beta$-sitosterol, stigmasterol and phytol from the leaves of A. camansi, while the stems yielded polyprenol, cycloartenol and cycloartenol acetate (Tsai et al., 2013). In another study, the leaves of A. altilis yielded $\beta$-sitosterol, triglycerides, squalene, polyprenol, lutein and fatty acids, while A. odoratissimus afforded $\beta$-sitosterol, triglycerides and fatty acids from the flesh of the fruit and seeds; and $\beta$-sitosterol, fatty acids and hydrocarbons from the fruit rind (Ragasa et al., 2014). Furthermore, the unripe fruit of $A$. heterophyllus afforded cycloartenone, cycloartenol, and a diastereomeric mixture of 2,3-butanediols (Ragasa and Jorvina, 2004).

We report herein the fractionation by silica gel chromatography of the dichloromethane extract of the twigs of $A$. ovatus. The following compounds from the twigs were identified by NMR spectroscopy: lupeol (1a), $\alpha$-amyrin (1b), $\beta$-amyrin (1c), lupeol fatty acid ester (2a), $\alpha$-amyrin fatty acid ester (2b), $\beta$-amyrin fatty acid ester (2b), betulin (3a), 3 $\beta, 28$-dihydroxyolean-12-ene (3b), oleanolic acid (4), $\beta$-sitosterol (5), and chlorophyll a (6) (Fig. 1). To the best of our knowledge this is the first report on the identification of these compounds from A. ovatus. 


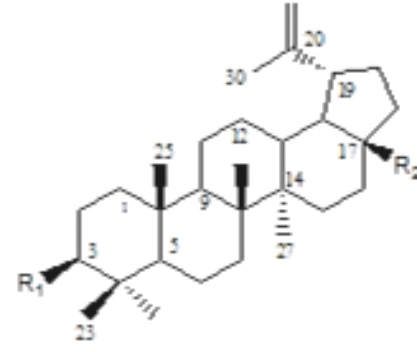

la $\mathrm{R}_{1}=\mathrm{OH}, \mathrm{R}_{2}=\mathrm{CH}_{3}$ 2a $\mathrm{R}_{1}=$ fatty acid, $\mathrm{R}_{2}=\mathrm{CH}_{3}$ 3a $\mathrm{R}_{1}=\mathrm{OH}, \mathrm{R}_{2}=\mathrm{CH}_{2} \mathrm{OH}$

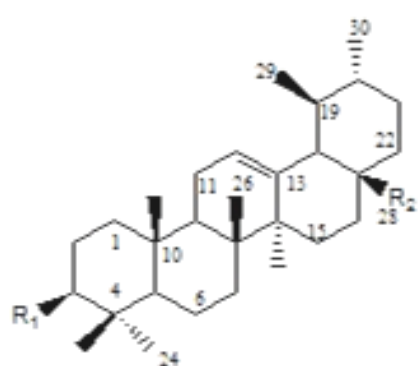

lb $\mathrm{R}_{1}=\mathrm{OH}, \mathrm{R}_{2}=\mathrm{CH}_{3}$ 2b $\mathrm{R}_{1}=$ fatty acid, $\mathrm{R}_{2}=\mathrm{CH}_{3}$

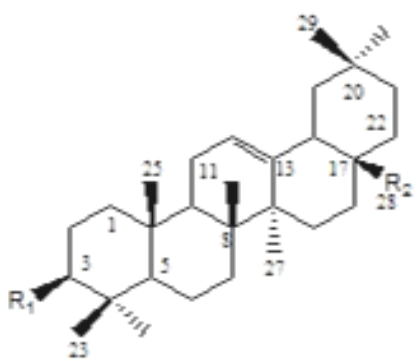

lc $\mathrm{R}_{1}=\mathrm{OH}, \mathrm{R}_{2}=\mathrm{CH}_{3}$ 2c $\mathrm{R}_{1}=$ fatry acid, $\mathrm{R}_{2}=\mathrm{CH}_{3}$ $3 \mathrm{~b} \mathrm{R}_{1}=\mathrm{OH}, \mathrm{R}_{2}=\mathrm{CH}, \mathrm{OH}$ $4 \mathrm{R}_{1}=\mathrm{OH}_{2} \mathrm{R}_{2}=\mathrm{COOH}$
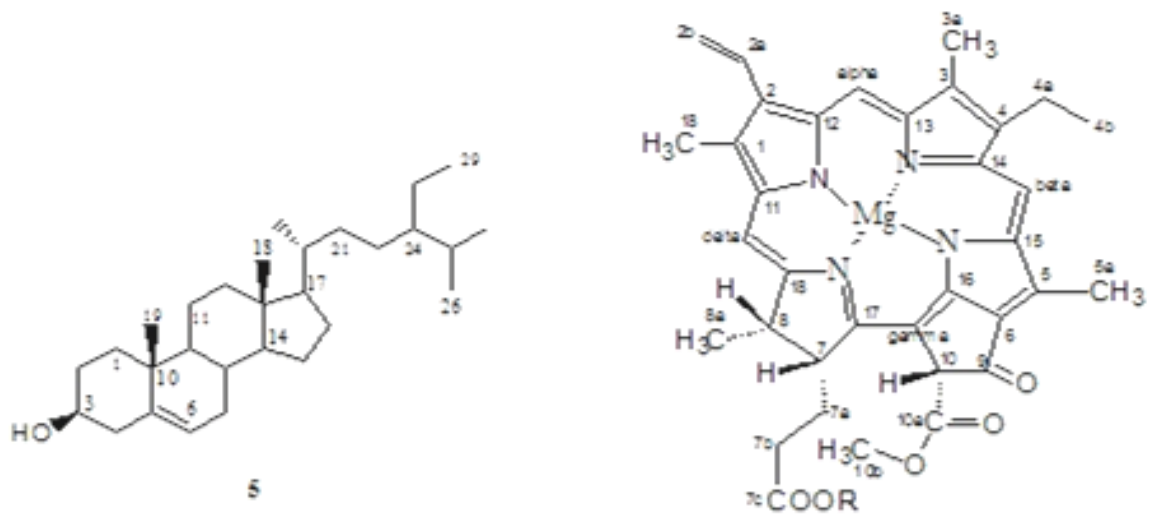

Fig. 1: Chemical constituents of the twigs of Artocarpus ovatus: lupeol (1a). $\alpha$-amvrin (1b). B-amvrin (1c). lubeol fattv acid ester (2a). $\alpha$-amvrin fattv acid ester

\section{MATERIALS AND METHODS}

General Experimental Procedure

NMR spectra were recorded on a Varian VNMRS spectrometer in $\mathrm{CDCl}_{3}$ at $600 \mathrm{MHz}$ for ${ }^{1} \mathrm{H} \mathrm{NMR}$ and $150 \mathrm{MHz}$ for ${ }^{13} \mathrm{C}$ NMR spectra. Column chromatography was performed with silica gel 60 (70-230 mesh). Thin layer chromatography was performed with plastic backed plates coated with silica gel $\mathrm{F}_{254}$ and the plates were visualized by spraying with vanillin $/ \mathrm{H}_{2} \mathrm{SO}_{4}$ solution followed by warming.

\section{Sample Collection}

The sample was collected from Bataan, Philippines in October 2013. It was identified as Artocarpus ovatus Blanco at the Jose Vera Santos Herbarium, Institute of Biology, University of the Philippines, Diliman, Quezon City.

\section{General Isolation Procedure}

A glass column 18 inches in height and 1.0 inche internal diameter was packed with silica gel. The crude extract from the twigs were fractionated by silica gel chromatography using increasing proportions of acetone in dichloromethane (10\% increment) as eluents. Fifty milliliter fractions were collected.
All fractions were monitored by thin layer chromatography. Fractions with spots of the same $R f$ values were combined and rechromatographed in appropriate solvent systems until TLC pure isolates were obtained. A glass column 12 inches in height and 0.5 inch internal diameter was used for the rechromatography. Two milliliter fractions were collected. Final purifications were conducted using Pasteur pipettes as columns. One milliliter fractions were collected.

\section{Isolation}

The air-dried twigs of A. ovatus (278.7 g) were ground in a blender, soaked in $\mathrm{CH}_{2} \mathrm{Cl}_{2}$ for 3 days and then filtered. The solvent was evaporated under vacuum to afford a crude extract $(8.1 \mathrm{~g})$ which was chromatographed using increasing proportions of acetone in $\mathrm{CH}_{2} \mathrm{Cl}_{2}$ at $10 \%$ increment. The $10 \%$ acetone in $\mathrm{CH}_{2} \mathrm{Cl}_{2}$ fraction was rechromatographed $(5 \times)$ in $5 \%$ EtOAc in petroleum ether to afford a mixture of $2 \mathbf{a}-2 \mathbf{c}(5 \mathrm{mg})$ after washing with petroleum ether. The $20 \%$ acetone in $\mathrm{CH}_{2} \mathrm{Cl}_{2}$ fraction was rechromatographed $(4 \times)$ in $15 \%$ EtOAc in petroleum ether to afford a mixture of 1a-1c (1 mg) after washing with petroleum ether. The $30 \%$ acetone in $\mathrm{CH}_{2} \mathrm{Cl}_{2}$ fraction was rechromatographed 
$(3 \times)$ in $20 \%$ EtOAc in petroleum ether to afford $5(6 \mathrm{mg})$ after washing with petroleum ether. The $40 \%$ acetone in $\mathrm{CH}_{2} \mathrm{Cl}_{2}$ fraction was rechromatographed $(3 \times)$ in $20 \%$ EtOAc in petroleum ether to afford a mixture of $\mathbf{3} \mathbf{a}$ and $\mathbf{3 b}(2 \mathrm{mg})$ after washing with petroleum ether. The 50\% acetone in $\mathrm{CH}_{2} \mathrm{Cl}_{2}$ fraction was rechromatographed $(4 \times)$ in $\mathrm{CH}_{3} \mathrm{CN}: \mathrm{Et}_{2} \mathrm{O}: \mathrm{CH}_{2} \mathrm{Cl}_{2}$ (1:1:8) by volume ratio to afford $\mathbf{4}$ $(4 \mathrm{mg}$ ) after washing with petroleum ether. The $60 \%$ acetone in $\mathrm{CH}_{2} \mathrm{Cl}_{2}$ fraction was rechromatographed (4x) in $\mathrm{CH}_{3} \mathrm{CN}: \mathrm{Et}_{2} \mathrm{OCH}_{2} \mathrm{Cl}_{2}(1: 1: 8)$ by volume ratio to afford $6(5 \mathrm{mg})$ after washing with petroleum ether, followed by $\mathrm{Et}_{2} \mathrm{O}$.

\section{Lupeol (1a)}

colorless solid. ${ }^{1} \mathrm{H}$ NMR $\left(600 \mathrm{MHz}, \mathrm{CDCl}_{3}\right):{ }^{1} \mathrm{H}$ NMR $\left(\mathrm{CDCl}_{3}, 500 \mathrm{MHz}\right) \delta 4.68(\mathrm{H}-29 \mathrm{~b}), 4.55(\mathrm{H}-29 \mathrm{a}), 3.18(\mathrm{H}-3), 1.68$ (s, $\left.\mathrm{H}_{3}-30\right), 0.96$ (s, $\left.\mathrm{H}_{3}-23\right), 0.78$ ( $\left.\mathrm{s}, \mathrm{H}_{3}-24\right), 0.83$ ( $\left.\mathrm{s}, \mathrm{H}_{3}-25\right), 0.94$ (s, $\left.\mathrm{H}_{3}-26\right), 1.06$ (s, $\left.\mathrm{H}_{3}-27\right), 0.91$ (s, $\left.\mathrm{H}_{3}-28\right), 1.68$ ( $\mathrm{s}, \mathrm{H}_{3}-30$ ).

\section{$\alpha$-Amyrin (1b)}

Colorless solid, ${ }^{1} \mathrm{H}$ NMR $\left(600 \mathrm{MHz}, \mathrm{CDCl}_{3}\right)$ : ${ }^{1} \mathrm{H}$ NMR $\left(\mathrm{CDCl}_{3}, 500 \mathrm{MHz}\right) \delta 3.15(\mathrm{H}-3), 0.67(\mathrm{H}-5), 5.15(\mathrm{H}-12), 0.94$ (s, $\mathrm{H}_{3}-23$ ), 0.76 (s, $\left.\mathrm{H}_{3}-24\right), 0.75$ (s, $\left.\mathrm{H}_{3}-25\right), 0.89$ (s, $\left.\mathrm{H}_{3}-26\right), 1.02$ (s, $\left.\mathrm{H}_{3}-27\right), 0.94$ (s, $\left.\mathrm{H}_{3}-28\right), 0.85$ (d, $\left.J=6.0 \mathrm{~Hz}, \mathrm{H}_{3}-29\right), 0.74$ (d, $J=$ $7.0 \mathrm{~Hz}, \mathrm{H}_{3}-30$ ).

\section{B-Amyrin (1c)}

Colorless solid. ${ }^{1} \mathrm{H}$ NMR $\left(600 \mathrm{MHz}, \mathrm{CDCl}_{3}\right)$ : ${ }^{1} \mathrm{H}$ NMR $\left(\mathrm{CDCl}_{3}, 500 \mathrm{MHz}\right) \delta 3.15$ (H-3), 0.67 (H-5), 5.22 (H-12), 0.77 (s, $\mathrm{H}_{3}-23$ ), 0.90 (s, $\mathrm{H}_{3}-24$ ), 0.74 (s, $\mathrm{H}_{3}-25$ ), 0.94 (s, $\left.\mathrm{H}_{3}-26\right), 1.16$ (s, $\left.\mathrm{H}_{3}-27\right), 1.06$ (s, $\left.\mathrm{H}_{3}-28\right), 0.86$ (s, $\left.\mathrm{H}_{3}-29\right), 0.79$ ( $\mathrm{s}, \mathrm{H}_{3}-30$ ).

\section{Lupeol fatty acid ester (2a)}

Colorless solid. ${ }^{13} \mathrm{C}$ NMR $\left(150 \mathrm{MHz}, \mathrm{CDCl}_{3}\right): 38.62$ (C1), 23.42 (C-2), 80.49 (C-3), 38.61 (C-4), 55.48 (C-5), 18.23 (C6), 34.71 (C-7), 40.97 (C-8), 50.99 (C-9), 38.62 (C-10), 21.06 (C11), 25.17 (C-12), 38.62 (C-13), 42.64 (C-14), 27.49 (C-15), 35.49 (C-16), 43.95 (C-17), 48.36 (C-18), 47.90 (C-19), 150.89 (C-20), 29.36 (C-21), 39.94 (C-22), 27.49 (C-23), 16.93 (C-24), 14.82 (C27), 18.23 (C-28), 109.39 (C-29), 19.35 (C-30), 173.69 (C-1'), 34.87 (C-2'), 31.92 (C-3'), 22.69, 25.17, 29.18-29.70 $\left(\mathrm{CH}_{2}\right)_{\mathrm{n}}, 14.12$ $\left(\mathrm{CH}_{3}\right)$.

\section{$\alpha$-Amyrin fatty acid ester (2b)}

Colorless solid. ${ }^{13} \mathrm{C}$ NMR $\left(150 \mathrm{MHz}, \mathrm{CDCl}_{3}\right): 38.68(\mathrm{C}-$ 1), 22.69 (C-2), 80.49 (C-3), 37.14 (C-4), 55.48 (C-5), 18.37 (C6), 32.48 (C-7), 39.68 (C-8), 47.89 (C-9), 37.11 (C-10), 23.31 (C11), 124.66 (C-12), 138.73 (C-13), 42.17 (C-14), 28.70 (C-15), 26.56 (C-16), 33.75 (C-17), 59.04 (C-18), 39.59 (C-19), 39.68 (C20), 31.25 (C-21), 41.51 (C-22), 28.06 (C-23), 16.92 (C-26), 23.42 (C-27), 28.06 (C-28), 17.46 (C-29), 21.38 (C-30), 173.69 (C-1'), 34.87 (C-2'), 31.92 (C-3'), 22.69, 25.17, 29.18-29.70 $\left(\mathrm{CH}_{2}\right)_{\mathrm{n}}, 14.12\left(\mathrm{CH}_{3}\right)$.

\section{$\beta$-Amyrin fatty acid ester (2c)}

Colorless solid. ${ }^{13} \mathrm{C}$ NMR $\left(\mathrm{CDCl}_{3}\right): 38.62(\mathrm{C}-1), 22.69$
(C-2), 80.49 (C-3), 37.14 (C-4), 55.48 (C-5), 18.37 (C-6), 32.48 (C-7), 39.68 (C-8), 47.22 (C-9), 37.14 (C-10), 23.66 (C-11), 121.95 (C-12), 144.30 (C-13), 41.51 (C-14), 26.08 (C-15), 26.91 (C-16), 32.48 (C-17), 47.83 (C-18), 46.76 (C-19), 31.25 (C-20), 34.87 (C-21), 37.14 (C-22), 28.06 (C-23), 16.92 (C-24), 16.92 (C26), 26.08 (C-27), 28.06 (C-28), 33.31 (C-29), 23.66 (C-30), 173.69 (C-1'), 34.87 (C-2'), 31.92 (C-3'), 22.69, 25.17, 29.18-29.70 $\left(\mathrm{CH}_{2}\right)_{\mathrm{n}}, 14.12\left(\mathrm{CH}_{3}\right)$.

\section{Betulin (3a)}

Colorless solid. ${ }^{1} \mathrm{H}$ NMR $\left(600 \mathrm{MHz}, \mathrm{CDCl}_{3}\right): \delta{ }^{1} \mathrm{H}$ NMR $\left(\mathrm{CDCl}_{3}, 500 \mathrm{MHz}\right) \delta 3.18(\mathrm{H}-3), 0.96\left(\mathrm{~s}, \mathrm{H}_{3}-23\right), 0.74$ (s, $\left.\mathrm{H}_{3}-24\right)$, 0.81 (s, $\left.\mathrm{H}_{3}-25\right), 0.98$ (s, $\left.\mathrm{H}_{3}-26\right), 1.00$ (s, $\left.\mathrm{H}_{3}-27\right), 3.33$ (d, H-28a), 3.79 (d, H-28b), 4.55 (d, H-29a), (d, $4.68 \mathrm{H}-29 \mathrm{~b}), 1.66$ (s, $\mathrm{H}_{3}-30$ ).

\section{3ק, 28-Dihydroxyolean-12-ene (3b)}

Colorless solid. ${ }^{1} \mathrm{H}$ NMR $\left(600 \mathrm{MHz}, \mathrm{CDCl}_{3}\right): \delta{ }^{1} \mathrm{H}$ NMR $\left(\mathrm{CDCl}_{3}, 500 \mathrm{MHz}\right) \delta 3.18(\mathrm{H}-3), 5.18$ (d, H-12), 0.96 (s, $\mathrm{H}_{3}-23$ ), 0.74 (s, $\left.\mathrm{H}_{3}-24\right), 0.87$ (s, $\mathrm{H}_{3}-25$ ), 0.98 (s, $\left.\mathrm{H}_{3}-26\right), 1.15$ (s, $\left.\mathrm{H}_{3}-27\right), 0.95\left(\mathrm{H}_{3}-29\right), 0.88\left(\mathrm{H}_{3}-29\right), \quad 3.20(\mathrm{~d}, \mathrm{H}-28 \mathrm{a}), 3.52(\mathrm{~d}, \mathrm{H}-$ 28b).

\section{Oleanolic acid (4)}

Colorless solid. ${ }^{1} \mathrm{H}$ NMR $\left(600 \mathrm{MHz}, \mathrm{CDCl}_{3}\right): \delta{ }^{1} \mathrm{H}$ NMR $\left(\mathrm{CDCl}_{3}, 500 \mathrm{MHz}\right) \delta 3.20(\mathrm{dd}, J=4.2,11.4 \mathrm{~Hz}, \mathrm{H}-3 \alpha), 5.26(\mathrm{t}, J=$ $3.6 \mathrm{~Hz}, \mathrm{H}-12), 2.81$ (dd, $J=4.2,13.8 \mathrm{~Hz}, \mathrm{H}-18), 0.96$ (s, $\mathrm{H}_{3}-23$ ), 0.73 (s, $\left.\mathrm{H}_{3}-24\right), 0.89$ (s, $\left.\mathrm{H}_{3}-25\right), 0.75$ ( $\left.\mathrm{s}, \mathrm{H}_{3}-26\right), 1.11$ (s, $\mathrm{H}_{3}-27$ ), $0.91\left(\mathrm{~s}, \mathrm{H}_{3}-29\right), 0.88\left(\mathrm{~s}, \mathrm{H}_{3}-30\right) ;{ }^{13} \mathrm{C}$ NMR $\left(150 \mathrm{MHz}, \mathrm{CDCl}_{3}\right): \delta$ 38.38 (C-1), 27.17 (C-2), 77.02 (C-3), 38.74 (C-4), 55.20 (C-5), 18.28 (C-6), 32.59 (C-7), 39.25 (C-8), 47.62 (C-9), 37.07 (C-10), 23.38 (C-11), 122.63 (C-12), 143.58 (C-13), 41.57 (C-14), 27.67 (C-15), 22.90 (C-16), 46.51 (C-17), 40.96 (C-18), 45.86 (C-19), 30.67 (C-20), 33.78 (C-21), 32.42 (C-22), 28.09 (C-23), 15.53 (C24), 15.31 (C-25), 17.12 (C-26), 25.93 (C-27), 183.29 (C-28), 33.06 (C-29), 23.56 (C-30).

\section{$\boldsymbol{\beta}$-Sitosterol (5)}

${ }^{1} \mathrm{H}$ NMR $\left(600 \mathrm{MHz}, \mathrm{CDCl}_{3}\right): \delta 3.51(\mathrm{~m}, \mathrm{H}-3), 2.26,2.21$ $\left(\mathrm{H}_{2}-4\right), 5.33 \mathrm{dd}(J=3.0,2.4 \mathrm{~Hz}, \mathrm{H}-6), 0.66$ (s, $\left.\mathrm{H}_{3}-18\right), 0.99$ (s, $\mathrm{H}_{3}-$ 19), 0.90 (d, $\left.J=6.6 \mathrm{~Hz}, \mathrm{H}_{3}-21\right), 0.79$ (d, $\left.J=7.2 \mathrm{~Hz}, \mathrm{H}_{3}-26\right), 0.82$ (d, $\left.J=6.6 \mathrm{~Hz}, \mathrm{H}_{3}-27\right), 0.85$ (t, $J=7.8 \mathrm{~Hz}, \mathrm{H}_{3}-29$ ).

\section{Chlorophyll a (6)}

${ }^{1} \mathrm{H}$ NMR $\left(600 \mathrm{MHz}, \mathrm{CDCl}_{3}\right): \delta 3.39$ (s, H-1), 7.98 (dd, $J$ $=18,12 \mathrm{~Hz}, \mathrm{H}-2 \mathrm{a}), 6.26(\mathrm{dd}, J=18,1.2 \mathrm{~Hz}, \mathrm{H}-2 \mathrm{~b}), 6.26(\mathrm{dd}, J=$ $12,1.2 \mathrm{~Hz}, \mathrm{H}-2 \mathrm{~b}$ ), 3.22 (s, H-3a), 3.67 (s, H-4a), 1.69 (t, $J=7.2$, H-4b), 3.76 (s, H- $1 \alpha$ or H-5 $\alpha$ ), 4.17 (m, H-7), 2.15, 2.46 (m, $\mathrm{H}_{2-}$ 7a), 2.32, $2.60\left(\mathrm{~m}, \mathrm{H}_{2}-7 \mathrm{~b}\right), 4.42(\mathrm{~m}, \mathrm{H}-8), 1.78\left(\mathrm{~d}, J=7.2 \mathrm{~Hz}, \mathrm{H}_{3}\right.$ 8a), 6.24 (s, H-10), 3.86 (s, $\left.\mathrm{H}_{3}-10 \mathrm{~b}\right), 9.38$ (s, H- $\alpha$ or H- $\beta$ ), 9.51 (s, $\mathrm{H}-\alpha$ or $\mathrm{H}-\beta), 8.54$ (s, H- $\delta$ ), 4.46 (m, P1), 5.11 (t, $J=1.2 \mathrm{~Hz}, \mathrm{P} 2$ ), 1.56 (br s, P17), 0.82 (d, $J=6.6 \mathrm{~Hz}, \mathrm{P} 18$ and P19), 0.76 (d, $J=$ $6.6 \mathrm{~Hz}, \mathrm{P} 16), 0.74$ (d, $J=6.6 \mathrm{~Hz}, \mathrm{P} 20)$. 


\section{RESULTS AND DISCUSSION}

Silica gel chromatography of the dichloromethane extract of the twigs of A. ovatus afforded lupeol (1a) (Prakash and Prakash), $\alpha$-amyrin (1b) (Raga et al., 2011a), $\beta$-amyrin (1c) (Raga et al., 2011a), lupeol fatty acid ester (2a) (Raga et al., 2011b), $\alpha$ amyrin fatty acid ester (2b) (Miranda et al., 2006), $\beta$-amyrin fatty acid ester (2c) (Barreiros et al., 2012), betulin (3a) (Tijjani et al., 2012), 3 $\beta$, 28-Dihydroxyolean-12-ene (3b) (Rahman et al., 2007), oleanolic acid (4) (Ragasa and Lim, 2005), $\beta$-sitosterol (5) (Cayme and Ragasa, 2004), and chlorophyll a (6) (Ragasa and de Jesus, 2014). The structures of 1-6 were identified by comparison of their ${ }^{1} \mathrm{H}$ and/or ${ }^{13} \mathrm{C}$ NMR data with those reported in the literature.

Although bioassays were not conducted on the isolated compounds, there were previous studies that reported on their biological activities. Lupeol (1a) exhibited antiurolithiatic and diuretic activity (Vidya et al., 2002). It prevented the formation of vesical calculi and reduced the size of the preformed stones in rats (Anand et al. 1994). $\alpha$-Amyrin (1b) and $\beta$-amyrin (1c) were reported to possess anti-inflammatory activity (Recio et al. 1995; Madeiros et al. 2007), while $\alpha$-amyrin, $\beta$-amyrin, and the 3-O-acyl derivatives of $\alpha$-amyrin (2b) and $\beta$-amyrin (2c) exhibited analgesic property (Otuki et al. 2005; Soldi et al. 2008).

Betulin (3a) inhibited cell growth and induction of apoptosis in human melanoma cell line (Orchel et al., 2014). It also induces apaptosis of Leishmania donovani. Treatment of promastigotes with betulin led to mitochondrial membrane damage, activation of caspase-like proteases, and DNA fragmentation in L. donovani promastigotes (Saudagar and Dubey, 2014).

An earlier study reported that ursolic acid and oleanolic acid (4) exhibited anti-tumor activity against human colon carcinoma cell line HCT15 with ursolic acid showing stronger activity than oleanolic acid ( $\mathrm{Li}$ et al., 2002). It exhibited antiinflammatory effects by inhibiting hyperpermeability, the expression of CAMs, and the adhesion and migration of leukocytes (Lee et al., 2013). It showed anti-inflammatory activities through the inhibition of the HMGB1 signaling pathway (Yang et al., 2012). It has anti-inflammatory, hepatoprotective, gastroprotective, anti-ulcer, and immunoregulatory effect (Vachalkova et al., 2004), gastroprotective effect on experimentally induced gastric lesions in rats and mice (Astudillo et al., 2002), inhibits mouse skin tumor (Oguro ,1998), protects against hepatotoxicants and is used in China to treat hepatitis (Lui et al., 1993), and significant antitumor activity on human colon carcinoma cell line HCT 15 (Li et al., 2002).

$\beta$-Sitosterol (5) was observed to have growth inhibitory effects on human breast MCF-7 and MDA-MB-231 adenocarcinoma cells (Awad et al., 2007). It was shown to be effective for the treatment of benign prostatic hyperplasia (Jayaprakasha et al., 2007). It was also reported to attenuate $\beta$ catenin and PCNA expression, as well as quench radical in-vitro, making it a potential anticancer drug for colon carcinogenesis (Baskar et al., 2010). It can inhibit the expression of NPC1L1 in the enterocytes to reduce intestinal cholesterol uptake (Jesch et al, 2009). It was reported to induce apoptosis mediated by the activation of ERK and the downregulation of Akt in MCA-102 murine fibrosarcoma cells (Moon et al., 2007).

Chlorophyll (6) and its various derivatives are used in traditional medicine and for therapeutic purposes (Edwards, 1954). Natural chlorophyll and its derivatives have been studied for wound healing (Kephart, 1955), anti-inflammatory properties (Larato and Pfao, 1970), control of calcium oxalate crystals (Tawashi et al., 1980), utilization as effective agents in photodynamic cancer therapy (Sternberg et al., 1998; Nourse et al., 1988; Henderson et al., 1997), and chemopreventive effects in humans (Egner et al., 2001; Egner et al., 2003). A review on digestion, absorption and cancer preventive activity of dietary chlorophyll has been provided (Hardwick et al., 1997).

Thus, the dichloromethane extract of the twigs of Artocarpus ovatus afforded compounds (1-6) with diverse biological activities.

\section{REFERENCES}

Anand R, Patnaik GK, Kulshreshtha DK, Dhawan N. Antiurolithiatic activity of lupeol, the active constituent of Crateva nuriala. Phytother Res 1994; 8(7):417-421.

Awad AB, Chinnman M, Fink CS, Bradford PG. $\beta$-Sitosterol activates Fas signaling in human breast cancer cells. Phytomed 2007; 14:747-754

Barreiros ML, David JM, Pereira PA, Guedes MLS, David JP. (2002). Fatty acid esters of triterpenes from Erythroxylum passerinum. J Braz Chem Soc 2002; 13:669-673.

Baskar AA, Ignacimuthu S, Paulraj G, Numair K. Chemopreventive potential of $\beta$-sitosterol in experimental colon cancer model - an In vitro and In vivo study. BMC Comp Alt Med 2010; 10: 24.

Cayme J-MC, Ragasa CY. Structure elucidation of stigmasterol and $\beta$-sitosterol from Sesbania grandiflora Linn. Pers. and $\beta$-Carotene from Heliotropium indicum Linn. by NMR Spectroscopy. Kimika 2004; 20(1/2):5-12.

De la Cruz Jr FS. Artocarpus odoratissimus Blanco. 1991. In Plant Resources of South-East Asia. 2. Edible Fruits and Nuts. E.W.M. Verheij and R.E. Coronel (eds.). PROSEA, Pudoc, Wageningen. 94-96.

Edwards BJ. Treatment of chronic leg ulcers with ointment containing soluble chlorophyll. Physiother 1954; 40:177-179.

Egner PA, Munoz A, Kensler TW. Chemoprevention with chlorophyllin in individuals exposed to dietary aflatoxin. Mutat Res 2003; 52(3):209-216.

Egner PA, Wang JB, Zhu YR, Zhang BC, Wu Y, Zhang QN. Chlorophyllin intervention reduces aflatoxin-DNA adducts in individuals at high risk for liver cancer. Proc Natl Acad Sci 2001; 98(25):1401-1406.

Fern K. 2012. Useful Tropical Plants Database.

Hardwick SJ, Carpenter KH, Law NS, Van Der Veen C, Marchant CE, Hird R, Mitchinson MJ. Free Radic Res 1997; 26 (4):351362.

Henderson BW, Bellnier DA, Greco WR, Sharma A, Pandry RK, Vaughan LA. An in Vivo Quantitative Structure-Activity Relationship for a Congeneric Series of Pyropheophorbide Derivatives as Photosensitizers for Photodynamic Therapy. Cancer Res 1997; 57:40004007.

Jagtap UB, Bapat VA, Artocarpus: a review of its traditional uses, phytochemistry and pharmacology. J Ethnopharmacol 2010; 129(2):142-66.

Jayaprakasha GK, Mandadi KK, Poulose SM, Jadegoud Y, Gowda GA, Patil BS. Inhibition of colon cancer growth and antioxidant activity of bioactive compounds from Poncirus trifoliate (L.) Raf. Bioorg Med Chem, 2007; 15:4923-4932. 
Jesch ED, Seo JM, Carr TP, Lee JY. Sitosterol reduces messenger RNA and protein expression levels of Niemann-Pick C1-like 1 in FHs 74 Int cells. Nutr Res 2009; 29(12):859-66.

Kephart JC. Chlorophyll derivatives - their chemistry, commercial preparation and uses. Econ Bot 1955; 9:3-18.

Larato DC, Pfao FR. Effects of a water-soluble chlorophyllin ointment on gingival inflammation. N Y State. Dent J 1970; 36:291-293.

Lee W, Yang EJ, Ku SK, Song KS, Bae JS. Anti-inflammatory effects of oleanolic acid on LPS-induced inflammation in-vitro and invivo. Inflammation 2013; 36(1):94-102.

Li J, Guo W-J, Yang Q-Y. Effects of ursolic acid and oleanolic acid on human colon carcinoma cell line HCT15. World J Gastroenterol 2002; 8(3):493-495.

Madeiros R, Otuki MF, Avellar MC, Calixto JB. 2007. Mechanisms underlying the inhibitory actions of the pentacyclic triterpene -amyrin in the mouse skin inflammation induced by phorbol ester 12-Otetradecanoylphorbol-13-acetate. Eur J Pharmacol 55(9):227-235.

Miranda RRS, Silva GDF, Duarte LP, Fortes ICP, Vieira Filho SA. Structural determination of $3 \beta$-stearoyloxy-urs-12-ene from Maytenus salicifolia by $1 \mathrm{D}$ and $2 \mathrm{D}$ NMR and quantitative ${ }^{13} \mathrm{C}$ NMR spectroscopy. Magnetic Resonance in Chemistry 2006; 44:127-131.

Moon DO, Kyeong Jun L, Yung HC, Gi-Young K. Moon DO, Kyeong Jun L, Yung HC, Gi-Young K. Int Immunopharmacol 2007; 7:1044-1053.

Nourse WL, Parkhurst RM, Skinner WA, Jordan RT. Photodynamic toxicity of porphyrins and chlorins for a human tumor cell line: combined light and concentration dose responses for the retained fraction. Biochem Biophys Res Commun 1988; 151:506-511.

Orchel A, Kulczycka A, Chodurek E, Bebenek E, Borkowska P, Boryczka S, Kowalski J, Dzierzewicz X. Influence of betulin and 28-Opropynoylbetulin on proliferation and apoptosis of human melanoma cells (G-361). Postepy Hig Med Dosw, 2014; 68(0):191-197.

Otuki C, Ferreira J, Lima F, Meyre-Silva C, Malheiros A, Muller L, Cani G, Santos A, Yunes R, Calixto J. Antinociceptive properties of a mixture of $\alpha$-amyrin and $\beta$-amyrin triterpenes: evidence for participation of protein kinase $\mathrm{C}$ and protein kinase A pathways. $\mathrm{J}$ Pharmacol Exp Therapeutics 2005; 31(1):310-318.

Prakash CVS, Prakash I. Isolation and Structural Characterization of Lupane Triterpenes from Polypodium vulgare. Res J Pharm Sci 2012; 1(1):23-27.

Raga DD, Alimboyoguen AB, Shen C-C, Ragasa CY. Triterpenoids and an anti-angiogenic sterol from Ardisia pyramidalis Cav. Pers. Philipp Agric Scient 2011a; 94(2):103-110.

Raga DD, Cheng CLC, Lee KCIC, Olaziman WZP, De Guzman VJA, Shen C-C, Franco FC, Ragasa CY. Bioactivities of triterpenes and sterol from Syzygium samarangense. Z Naturforsch 2011b; 66c:235-244.

Ragasa CY, Ng VA, Park JH, Kim DW, Cornelio K, Shen C-C. Chemical Constituents of Artocarpus altilis and Artocarpus odoratissimus. Res J Pharm Biol Chem Sci 2014; 5(4):1081-1087.
Ragasa CY, Jorvina K, Rideout JA. Antimicrobial compounds from Artocarpus heterophyllus. Philipp J Sci 2004; 133(2):97-101.

Ragasa CY, Lim K . Secondary metabolites from Schefflera odorata Blanco. Philipp J Sci 2005; 134(1):63-67.

Ragasa CY, de Jesus J. Porphyrins and polyprenols from Macaranga tanarius. Res J Pharm Biol Chem Sci 2014; 5(3):701708.

Rahman MZ, Sultana SJ, Faruquee CF, Ferdous F, Rahman MS, Islam MS, Rashid MA. Phytochemical and biological investigations of Erythrina variegata. Saudi Pharm J 2007; 15(2):140145 .

Recio MC, Giner RM, Manez S, Rios JL. Structural requirements for the anti-inflammatory activity of natural triterpenoids. Planta Med 1995; 61(2):181-185.

Saudagar P, Dubey VK, Molecular mechanisms of in vitro betulin-induced apoptosis of Leishmania donovani Am J Trop Med Hyg 2014; 90(2):354-360.

Soldi C, Pizzolatti G, Luiz A, Marcon R, Meotti F, Miotob L, Santos A. Synthetic derivatives of the $\alpha$ - and $\beta$-amyrin triterpenes and their antinociceptive properties. Bioorganic and Medicinal Chemistry $2008 ; 16(6): 3377-3386$

Sternberg ED, Dolphin D, Bruckner C. Porphyrin-based Photosensitizers for Use in Photodynamic Therapy. Tetrahedron 1998; 54:4151-4152.

Tawashi R, Cousineau M, Sharkawi M. Effect of sodium copper chlorophyllin on the formation of calcium oxalate crystals in rat kidney. Invest Urol 1980; 18:90-92.

Tijjani A, Ndukwe IG, Ayo RG. Isolation and characterization of lup-20(29)-ene-3,28-diol (betulin) from the stem-bark of Adenium obesum (Apocynaceae). Trop J Pharm Res 2012; 11 (2):259-262.

Tsai P-W, de Castro-Cruz K, Shen C-C, Ragasa CY. Chemical constituents of Artocarpus camansi. Phcog J 2013; 5:80-82.

Vidya L, Leni M, Varalakshmi P. 2002. Evaluation of the effect of triterpenes on urinary risk factors of stone formation in pyridoxine hyperoxaluric rats. Phytother Res 16 (6):514-518.

Yang EJ, Lee W, Ku SK, Song KS, Bae JS. Anti-inflammatory activities of oleanolic acid on HMGB1 activated HUVECs. Food Chem Toxicol 2012; 50(5) 1288-94.

\section{How to cite this article:}

Consolacion Y. Ragasa, Jocelyn L. Caro, Chien-Chang Shen. Triterpenes and Sterol from Artocarpus ovatus. J App Pharm Sci, 2014; 4 (10): 007-011. 